\title{
Evaluation of Association between Vaginal Infections and High-Risk Human Papillomavirus Types in Female Sex Workers in Spain
}

\author{
C. Rodriguez-Cerdeira, ${ }^{1}$ E. Sanchez-Blanco, ${ }^{2}$ and A. Alba $^{3}$ \\ ${ }^{1}$ Dermatology Department, CHUVI and University of Vigo, Vigo, Spain \\ ${ }^{2}$ University of Vigo, Vigo, Spain \\ ${ }^{3}$ Spanish Society of Cervical Pathology and Colposcopy, Centre for Molecular and Cellular Studies, Lugo, Spain
}

Correspondence should be addressed to C. Rodriguez-Cerdeira, crodcer@uvigo.es

Received 9 April 2012; Accepted 13 June 2012

Academic Editors: V. Anaf and I. Figa-Talamanca

Copyright ( 92012 C. Rodriguez-Cerdeira et al. This is an open access article distributed under the Creative Commons Attribution License, which permits unrestricted use, distribution, and reproduction in any medium, provided the original work is properly cited.

\begin{abstract}
Background. Infection with and persistence of high-risk human papillomavirus (HR-HPV) are the strongest risk factors for cervical cancer. In addition, other genital microorganisms may also be involved in the progression of HPV-associated lesions. Objetive. To evaluate the association of the vaginal microbiota (Candida spp., Trichomonas vaginalis, and bacterial vaginosis) with HR-HPV infection in Spanish female sex workers (FSWs). Methods. This cross-sectional study involved 208 (FSWs; age, 18-49 years) who visited a sexually transmitted infection (STI) information and prevention center (SERGAS) between January 2010 and December 2011. Face-to-face interviews were carried out. Cervical and vaginal samples were examined for human papillomavirus (HPV), Trichomonas vaginalis, Candida spp., and microorganisms related to bacterial vaginosis (BV). Results. HR-HPV was found to be significantly associated with BV in FSWs with positive results for HPV16-related types (31, 33, 35, and 52). T. vaginalis was isolated in FSWs with the following HR-HPVs: 18, 45, 66, and 68. Candida spp. were isolated only in FSWs with HPV 18-positive infection. Conclusion. We demonstrate a significant prevalence of HR-HPVs in FSWs with disturbances in the vaginal microbiota.
\end{abstract}

\section{Introduction}

High-risk human papillomavirus (HR-HPV) infection is the most common sexually transmitted infection (STI) among young adult women and plays a critical role in the development of genital cancer. Recently, 15 HR oncological viral strains have been identified, including the HPV 16 group (alpha-9) of the alpha-papillomavirus genus (HPV 31, HPV33, HPV 35, HPV 52, and HPV 58) and the HPV 18 group (alpha-7; HPV39, HPV 45, HPV 59, and HPV 68) $[1,2]$. Biological susceptibility to HR-HPV acquisition and reduced immune competence for clearance of HR-HPV infection could result from common treatable vaginal infections that disrupt the intricately balanced vaginal ecosystem and its innate protective mechanisms against infection and disease [3].

Earlier reports have suggested a link between genital tract inflammation and cervical cancer, only a few studies have controlled for HR-HPV genotypes, and no study has examined this infection in female sex workers (FSWs). The association between self-reported abnormal vaginal discharge and cervical intraepithelial neoplasia (CIN) in HRHPV-infected women further suggests a link between genital tract anomalies and cervical cancer $[4,5]$. Bacterial vaginosis (BV) and trichomoniasis are associated with high levels of anaerobic microorganisms and their byproducts, which can damage the vaginal epithelium, degrade cervical mucus, and cleave immunoglobulin A [6-8], and the evidence suggests an important association between HR-HPV and alterations in the vaginal microbiome $[9,10]$. BV and trichomoniasis are categorized, along with vulvovaginal candidiasis, under the slight misnomer of vaginitis. Candida albicans is the most prevalent species in the majority of cases of asymptomatic colonization and vulvovaginal candidiasis. However, certain species of Candida are more pathogenic than others, induce hyphal and pseudohyphal formation, and enhance 
proteolytic activity and antigen modulation. These properties enable Candida to penetrate the mucosal surface and induce mucosal swelling, erythema, and exfoliation of cells [11]. In addition, some studies have reported that cervical cytologic abnormalities occur more often in women who have abnormal vaginal microbiota than in women without this condition. Therefore, we were interested in investigating whether women who carry Candida spp. are more prone to acquiring cervical cytologic abnormalities over time than women who have a known, disturbed, bacterial vaginal microbiota $[9,10]$.

Therefore, the purpose of the current study was to determine the prevalence of vulvovaginitis caused by Trichomonas vaginalis, Candida spp., or BV in a group of FSWs with HRHPV infection.

\section{Materials and Methods}

2.1. Sample Size. Approval for the study was obtained from the Ethics Committee of Galician (Spain) Human Research. HIV-negative FSWs (age, 18-49 years) who visited an STI information and prevention center in the north-eastern region of Spain between January 2010 and December 2011 were included in the study. The sample size was calculated to allow for a prevalence of $50 \%$ or higher for HR-HPV, with a confidence interval of $95 \%$ and an error of $6.5 \%$.

2.2. Statistical Analysis. The $\chi^{2}$ test was used for statistical analysis, while the odds ratio (OR) was used to measure the strength of the association between vaginal infections and high-risk human papillomavirus types. Statistical tests were considered significant if the $P$ value was $\leq 0.05$. Logistic regression analysis was used to assess the simultaneous effect of more than 1 variable on the risk of HPV infection and to identify possible confounding factors. We determined the OR by using nonconditional logistic regression but using the correct definition; we could not estimate the prevalence ratio (PR) because we did not have any patients who were not exposed to the infection. The software used for the statistical analysis was the Statistical Package for the Social Sciences (SPSS), version 18.0.1.

2.3. Detection of HR-HPV. Cervical samples were collected with a cervical brush, and all cervical swabs were placed a $1 \mathrm{~mL}$ a tube containing of the Digene specimen transport medium (Digene Corporation, Gaithersburg, MD, USA) and were stored at $-20^{\circ} \mathrm{C}$ until testing. The presence of HRHPV infection was determined through the Digene HRHPV test-Hybrid Capture II (HC2)-by using the HRHPV probe B cocktail, which identified types 16, 18, 31, $33,35,39,45,51,52,56,58,59$, and 68, in accordance with the manufacturer's instructions. To minimize the cost of testing, an HC2 HPV DNA test was performed for all samples with only the high-risk HPV probe mixture. Positive specimens were detected by binding of hybridization complexes onto the surface of the microplate wells coated with monoclonal antibodies specific to RNA-DNA hybrids. Immobilized hybrids were detected by the addition of an alkaline phosphatase-conjugated antibody to RNA-DNA hybrids, followed by the addition of a chemiluminescent substrate.

2.4. Detection of Bacterial Vaginosis. The presence of BV was evaluated microscopically in samples collected from the posterior vaginal fornix. BV was diagnosed on the basis of the presence of 3 of the following clinical and microscopic findings: vaginal $\mathrm{pH}$ greater than 4.5, presence of clue cells, grey homogenous vaginal discharge, and positive whiff test, in which a fishy odor is released after the addition of $10 \%$ potassium hydroxide solution to the vaginal fluid.

2.5. Detection of $T$. vaginalis. The vaginal swab was placed in $0.2 \mathrm{~mL}$ of sterile physiologic saline for wet mount evaluation and examined microscopically (400x) for motile T. vaginalis within $15 \mathrm{~min}$. Sample collection was performed using calcium alginate swabs, which were transported in the Stuart Amies transport medium (Copan Innovation), kept at room temperature, and sent to the laboratory within $6 \mathrm{~h}$ of collection for culture on Roiron medium (MAIM S.L.). T. vaginalis was first detected by direct microscopic visualization of the fresh sample, followed by culture of the vaginal discharge on the Roiron medium and aerobic incubation at $37^{\circ} \mathrm{C}$. The broth was examined microscopically for motile trichomonads on days 1,3 , and 5 of culture.

2.6. Detection of Candida spp. Samples were collected using calcium alginate swabs and transported in the Stuart Amies transport medium. The samples were kept in a $\mathrm{T}^{\circ}$ environment and then sent to the laboratory within $6 \mathrm{~h}$, as recommended by the manufacturer. Confirmatory microbiological culture of vaginal discharge was carried out in Sabouraud chromogenic medium (Candida ID2 (CAN2); bioMérieux) in the case of women for whom a positive diagnosis was suspected but for whom a negative result was obtained by direct visualization. For samples that did not test positive following microbiological culture, complementary diagnosis analysis using the Vitek ID-YST system (bioMérieux) was also performed.

\section{Results}

This cross-sectional study involved FSWs who visited an STI information and prevention center in Vigo, Spain, between January 2010 and December 2011. All FSWs who visited the center were invited to participate in the study, and the refusal rate was very low.

Finally, 208 HIV-negative FSWs aged 18-49 years were involved in this study. The mean age of the study population was 27 years, whilst $60 \%$ of participants belonged to the target age group of 18-25 years. HR-HPV prevalence peaked in younger women aged 18-25 years. As expected, the prevalence of $T$. vaginalis in FSWs was high (17\%). HR-HPV infection was present in 7.5-35.5\% of these women, and the peak prevalence of this infection occurred 20 years earlier than that for T. vaginalis. 
Data for HR-HPV-positive samples were grouped as belonging to HPV16-related genotypes $(31,33,35,52$, and 58), HPV18-related genotypes $(39,45,59$, and 68$)$, or other genotypes, including HPV51 and HPV66. In the study group of 208 FSWs, 81 women had positive results for HPV16 infection and 48 for HPV18 infection. The distribution of frequencies for different genotypes of HPV is shown in Table 1 [12].

Data from univariate analysis showed that BV was strongly associated with HR-HPV, as was the presence of $T$. vaginalis. We found no relationship between Candida spp. and HR-HPV, with the exception of HPV18 (Table 2).

Among women with vaginal T. vaginalis, Candida spp. was associated with HPV16 infection $(\mathrm{OR}=0.10,95 \% \mathrm{CI}$ : 0.002-0.048). Moreover, among women with BV, vaginal Candida spp.colonization was associated with HPV18 infections (Mantel and Haenszel OR $=0.030$, 95\% CI: 0.0110.083). When both vaginal Candida spp. and T. vaginalis were removed from the model, BV was still significantly associated with HR-HPV in all the FSWs. In contrast, when BV was removed from the model, vaginal Candida spp.colonization was not associated with HR-HPV, except for HPV18 (OR $=0.448,95 \%$ CI: 0.232-0.866). The presence of vaginal Candida spp.colonization alone was not significantly associated with all the HR-HPV genotypes, except for HPV18 (OR $=0.448,95 \%$ CI: 0.232-0.866). However, when Candida spp. colonization was present concurrently with BV or trichomoniasis, it negatively affected the outcomes for certain HPV types.

Three covariables were introduced into the multivariate logistical regression model. The distribution of $\mathrm{BV}$ in women with HR-HPV was statistically significant in HPV16 and HPV16-related genotypes (HPV31, 33, 35, and 52). Thus, we expect that, among the $16 \mathrm{HR}-\mathrm{HPV}$-negative participants, $88 \%$ did not have BV. This equates to $66 \%$ of HPV $31-$ negative, $82 \%$ of HPV33-negative, $75 \%$ of HPV35-negative, and $72 \%$ of HPV52-negative participants. In contrast, the presence of $T$. vaginalis was statistically significant in women with HPV18, HPV45, HPV66, or HPV68; the presence of Candida spp. was found to be statistically significant only when HPV18 was detected. This result was further associated with both Candida spp. and T. vaginalis (Table 3).

\section{Discussion}

According to Cheng et al. and Muñoz et al., HPV types may be organized according to (1) HPV16-related types $(31,33,35,52$, and 58) or HPV types belonging to the alpha-papillomavirus-9 species, (2) HPV18-related types $(39,45,59,68)$ or HPV types belonging to the alphapapillomavirus-7 species, or (3) others, including HPV51 and HPV66. We have used this classification system in our study $[1,13-16]$. BV is the main cause of abnormal vaginal discharge in women of reproductive age. It is characterized by replacement of lactobacilli by a mixture of anaerobic and aerobic bacteria (common agents include Gardnerella vaginalis, Mycoplasma hominis, and Ureaplasma urealyticum) [17]. In women without $\mathrm{BV}$, hydrogen peroxide-producing lactobacilli dominate vaginal microbiota [18]. The microorganisms responsible for BV increase the levels of mucindegrading enzymes, which may play a role in the degradation of the gel layer that coats the vaginal and cervical epithelium and endocervical mucus $[19,20]$. BV-associated microorganisms also produce cytokines and inflammatory mediators, which have been linked to pregnancy complications and may increase susceptibility to STIs [20]. Livengood et al. reported that $84 \%$ of BV-positive women also had positive results for sialidases [21]. Such enzymes may promote virulence by destroying the protective mucosal barrier and may hence increase susceptibility to cervical HR-HPV infection by facilitating adherence, invasion, and, eventually, incorporation of HPV-activated oncogenes into the genome of cells in the transformation zone. Abnormal vaginal microbiota may also be implicated in the maintenance of subclinical HR-HPV infection. Enzymes that are produced by anaerobic bacteria and are involved in the pathogenesis of BV can potentially alter immune signals and promote degradation of protective host factors, rendering women more susceptible to acquiring HR-HPV [22-25]. However, it is still not known whether BV and cervical HR-HPV infections are related because there is a biological symbiotic relationship between them or because both occur frequently in sexually active women. The role of sexual transmission in causing or promoting BV continues to be a topic of debate, as studies have shown that lesbians have a high prevalence of BV [26].

Candida spp. infection is the most prevalent mycosis in the majority of cases of asymptomatic colonization and vulvovaginal candidiasis. However, certain species of Candida are more pathogenic than the other species and induce hyphal and pseudohyphal formation and enhance proteolytic activity and antigen modulation [27]. These features enable Candida spp. to penetrate the mucosal surface and induce mucosal swelling, erythema, and cell exfoliation. The role of $T$. vaginalis and Candida spp. in HR-HPV pathogenesis has been evaluated by some authors, including Engberts et al. [28] who established that the presence of Candida spp. is not associated with an increased risk of acquiring HR-HPV.

In a study involving cytological analysis of samples obtained from 17,391 outpatients during January 1997August 1998 in Brazil, 390 samples (2.24\%) had alterations that were consistent with HPV infection and were sometimes associated with CIN I. The results showed that G. vaginalis was the most frequent bacterial agent found in women with HR-HPV infection $(23.6 \%$ versus $17.4 \%$; $P<0.05)$, while in the control group the most frequent agent was Candida spp. (23.9\% versus $13.8 \%$; $P<0.001)$ [29]. This result is in accordance with the findings of Wang and Lin [30] and Chakrabarti et al. [31], who also reported that there was no obvious association between the presence of Candida spp. and cytologic changes in the vagina. Candida spp. is not a cofactor in the presence of HR-HPV. However, it has been found that Candida spp. is more frequently found in women without cervical intraepithelial neoplasia (CIN) [32]. As reported by Watts et al. [33], T. vaginalis and vaginal Candida spp. colonizations were not associated with HRHPV infection in HIV-negative women. 
TABLE 1: Genotypes in human papillomavirus positive women.

\begin{tabular}{|c|c|c|c|c|c|c|c|c|}
\hline \multicolumn{9}{|c|}{ Prevalence of HPV } \\
\hline \multicolumn{3}{|c|}{ HPV 16-related types $(31,33,35,52$, and 58$)$} & \multicolumn{3}{|c|}{ HPV 18 -related types $(39,45,59,68)$} & \multicolumn{3}{|c|}{ HPV 51 Y 66} \\
\hline Type of HPV & $\begin{array}{c}\text { Number of sex } \\
\text { workers }\end{array}$ & $\%$ & Type of HPV & $\begin{array}{c}\text { Number of sex } \\
\text { workers }\end{array}$ & $\%$ & Type of HPV & $\begin{array}{c}\text { Number of sex } \\
\text { workers }\end{array}$ & $\%$ \\
\hline HPV 16 & 81 sex workers & $38.9 \%$ & HPV 18 & 48 sex workers & $23.1 \%$ & HPV 51 & 52 sex workers & $25 \%$ \\
\hline HPV 31 & 59 sex workers & $28.4 \%$ & HPV 39 & 45 sex workers & $21.6 \%$ & HPV 66 & 38 sex workers & $18.3 \%$ \\
\hline HPV 33 & 52 sex workers & $25 \%$ & HPV 45 & 38 sex workers & $18.3 \%$ & & & \\
\hline HPV 35 & 39 sex workers & $18.8 \%$ & HPV 56 & 50 sex workers & $24 \%$ & & & \\
\hline HPV 52 & 68 sex workers & $32.7 \%$ & HPV 59 & 23 sex workers & $11.1 \%$ & & & \\
\hline HPV 58 & 54 sex workers & $26 \%$ & HPV 68 & 31 sex workers & $14.9 \%$ & & & \\
\hline
\end{tabular}

*According to Muñoz et al. [12] and Li et al. [6]. 12 types were classified as high risk. 2 types as probably high risk.

TABLE 2: Characteristics of studied group: uivariate analysis.

\begin{tabular}{|c|c|c|c|c|c|c|}
\hline & \multicolumn{2}{|c|}{ Bacterial vaginosis } & \multicolumn{2}{|c|}{ Trichomonas vaginalis } & \multicolumn{2}{|c|}{ Candida spp. } \\
\hline & OR & 95\% confidence interval & OR & 95\% confidence interval & OR & 95\% confidence interval \\
\hline HPV 16 & 0.058 & $0.023-0.147$ & 0.083 & $0.033-0.213$ & 1.051 & $0.600-1.840$ \\
\hline HPV 18 & 0.078 & $0.036-0.171$ & 0.034 & $0.013-0.085$ & 0.448 & $0.232-0.866$ \\
\hline HPV 31 & 0.209 & $0.103-0.425$ & 0.199 & $0.0093-0.423$ & 1.292 & $0.701-2.381$ \\
\hline HPV 33 & 0.157 & $0.075-0.326$ & 0.207 & $0.097-0.443$ & 1.169 & $0.620-2.203$ \\
\hline HPV 35 & 0.122 & $0.056-0.266$ & 0.113 & $0.050-0.253$ & 1.232 & $0.608-2.496$ \\
\hline HPV 39 & 0.071 & $0.032-0.158$ & 0.026 & $0.010-0.067$ & 0.831 & $0.424-1.628$ \\
\hline HPV 45 & 0.246 & $0.122-0.542$ & 0.210 & $0.093-0.474$ & 0.644 & $0.311-1.337$ \\
\hline HPV 51 & 0.461 & $0.224-0.950$ & 0.376 & $0.176-0.803$ & 1.169 & $0.620-2.203$ \\
\hline HPV 52 & 0.168 & $0.082-0.347$ & 0.169 & $0.078-0.367$ & 1.166 & $0.650-2.090$ \\
\hline HPV 56 & 0.428 & $0.207-0.886$ & 0.350 & $0.163-0.750$ & 1.186 & $0.624-2.256$ \\
\hline HPV 58 & 0.256 & $0.126-0.522$ & 0.224 & $0.105-0.476$ & 1.573 & $0.832-2.971$ \\
\hline HPV 59 & 0.262 & $0.105-0.658$ & 0.197 & $0.077-0.504$ & 0.599 & $0.250-1.436$ \\
\hline HPV 66 & 0.022 & $0.008-0.058$ & 0.005 & $0.001-0.018$ & 1.023 & $0.504-2.074$ \\
\hline HPV 68 & 0.012 & $0.003-0.045$ & 0.021 & $0.007-0.060$ & 1.001 & $0.465-2.156$ \\
\hline
\end{tabular}

We have demonstrated a stronger association between bacterial vaginosis versus Trichomonas vaginales and high-risk human papillomavirus types.

TABLE 3: Multivariate analysis: odds ratio for prevalent high-risk human papillomavirus (HR-PV) among all FSWs.

\begin{tabular}{|c|c|c|c|c|c|c|}
\hline & \multicolumn{2}{|c|}{ Bacterial vaginosis } & \multicolumn{2}{|c|}{ Trichomonas vaginalis } & \multicolumn{2}{|c|}{ Candida spp. } \\
\hline & OR & 95\% confidence interval & OR & 95\% confidence interval & OR & $95 \%$ confidence interval \\
\hline HPV 16 & 0.117 & $0.038-0.359$ & - & - & - & - \\
\hline HPV 18 & - & - & 0.047 & $0.012-0.177$ & 3.423 & $1.3227-8.829$ \\
\hline HPV 31 & 0.342 & $0.125-0.939$ & - & - & - & - \\
\hline HPV 33 & 0.179 & $0.062-0.512$ & - & - & - & - \\
\hline HPV 35 & 0.244 & $0.080-0.740$ & - & - & - & - \\
\hline HPV 39 & - & - & - & - & - & - \\
\hline HPV 45 & - & - & 0.257 & $0.077-0.858$ & - & - \\
\hline HPV 51 & - & - & - & - & - & - \\
\hline HPV 52 & 0.281 & $0.104-0.763$ & - & - & - & - \\
\hline HPV 56 & - & - & - & - & - & - \\
\hline HPV 58 & - & - & - & - & - & - \\
\hline HPV 59 & - & - & - & - & - & - \\
\hline HPV 66 & - & - & 0.040 & $0.011-0.148$ & - & - \\
\hline HPV 68 & - & - & 4.968 & $1.353-18.250$ & - & - \\
\hline
\end{tabular}

We detect a significant effect of BV versus trichomoniasis on incident HR-HPV. 
In contrast to the finding for $\mathrm{BV}$ and $T$. vaginalis, it appears that the presence of Candida spp. in the vagina is not a cofactor in the development of cervical cancer. This is consistent with the hypothesis that the local cervicovaginal milieu plays a role in susceptibility to HR-HPV infection since women who carry Candida spp. are likely to possess a healthy Lactobacillus-predominated vaginal microbiota, in contrast to women with dysbacteriosis [34]. Only a limited number of studies have recognized an association between the presence of $T$. vaginalis and CIN or an association between T. vaginalis and HR-HPV infection [34]. These findings may be explained by common etiological factors and, in particular, by the number of sex partners, which is naturally difficult to prove with a high degree of certainty [34]. Several hypotheses have been proposed to explain the pathogenesis and virulence of $T$. vaginalis. One hypothesis is that the parasite can act as a potential catalyst in the development of secondary infections, including HR-HPV, by producing a wide array of enzymes such as cysteine proteinase enzymes that are linked with cytotoxicity and the degradation of basement membrane components. Furthermore, some studies have shown that the double-stranded RNA of $T$. vaginalis is also associated with differential expression of enzymes and may therefore affect virulence [35-39]. Therefore, it is also possible that $T$. vaginalis could alter the natural history of various sexually transmitted diseases and, in particular, HR-HPV, by increasing virulence. This study clearly shows that women with positive cytological results for $T$. vaginalis are at significant risk of acquiring HRHPV infection [40, 41].

In addition to the conventional STIs caused by Neisseria gonorrhoeae, Chlamydia trachomatis, and T. vaginalis, HRHPV infection has been found to be common (36\%), and oncogenic genotypes have been found in $44 \%$ of HR-HPVpositive patients [42].

In another study, T. vaginalis was found in 80 of the patients $(0.2 \%)$ [43]. Of these 80 patients, 57 were available for HR-HPV testing (age: 8 patients $<30$ years, 42 patients $30-50$ years, and 7 patients 50 years). As controls, high-risk HR-HPV was tested for in 150 patients with normal cytological features, from each of these 3 age categories. Women with T. vaginalis had a significantly higher prevalence of HR-HPV than women with normal results for cervical smear tests, irrespective of age $(P<0.01)$. Noël stated that these data suggest a potential association between these 2 infectious agents because of sexual intercourse and probably because of biochemical or immunological reasons [44]. Depuydt et al. concluded that the prevalence of $T$. vaginalis in Flanders is low, with only 3.7/1,000 women infected. HR-HPV is present in $15 \%$ of these women and the peak of its prevalence occurs 20 years earlier than that of detection of $T$. vaginalis. The present study design does not allow for a definitive answer for this paradoxical increase in T. vaginalis prevalence in the absence of HR-HPV in the age group of 40-50 years [44].

In our study, BV and T. vaginalis infection was common, with oncogenic genotypes (HPV18 and HPV18-related types 59 and 68 ) being present in $61 \%, 45.5 \%$, and $69 \%$ of positive samples. Moreover, T. vaginalis was present in $86 \%$ of HPV66-positive samples. These findings emphasize the importance of a cervical cancer prevention strategy, such as a cervical cytology screening program for HR-HPV, which is to be introduced soon.

\section{Conclusions}

This study suggests a positive association between BV and HR-HPVs (HPV 16, 31, 33, 35, and 52). Presence of $T$. vaginalis was statistically significant, in women with HPV 18 , 45,66 , and 68 . Candida spp. were not significantly associated with HR-HPV; they were more frequently among HR-HPVnegative women. These data confirm that screening for genital infections may reveal the simultaneous presence of different sexually transmitted microorganisms. These results suggest and emphasize the value of screening for genital infections in HR-HPV-positive patients in order to decrease the presence of other microorganisms and to reduce the probable synergistic effects of the vaginal microbiota. Prevention is important not only to avoid other STIs and their sequelae but also to reduce the influence of concomitant infection with other microorganisms on HR-HPV infection.

\section{Conflict of Interests}

The authors declare that they have no competing interests.

\section{Acknowledgments}

This paper was supported in part by the General Deputy for Research, Teaching, and Innovation (SERGAS, Xunta de Galicia, Spain).

\section{References}

[1] N. Li, S. Franceschi, R. Howell-Jones, P. J. F. Snijders, and G. M. Clifford, "Human papillomavirus type distribution in 30,848 invasive cervical cancers worldwide: variation by geographical region, histological type and year of publication," International Journal of Cancer, vol. 128, no. 4, pp. 927-935, 2011.

[2] C. Rodríguez-Cerdeira, A. Guerra-Tapia, R. Alcantara Caceres, and J. Escalas, "Human papilloma virus (HPV) and genital cancer," Open Dermatology Journal, vol. 3, pp. 111-122, 2009.

[3] S. J. Adad, R. V. de Lima, Z. T. Sawan et al., "Frequency of Trichomonas vaginalis, Candida sp and Gardnerella vaginalis in cervical-vaginal smears in four different decades," Sao Paulo Medical Journal, vol. 119, no. 6, pp. 200-205, 2001.

[4] M. G. Discacciati, J. A. Simões, E. S. Lopes et al., "Is bacterial vaginosis associated with squamous intraepithelial lesion of the uterine cervix?” Diagnostic Cytopathology, vol. 34, no. 5, pp. 323-325, 2006.

[5] G. Venegas, G. Boggiano, and E. Castro, "Prevalence of bacterial vaginosis in Chilean sex workers," Revista Panamericana de Salud Publica, vol. 30, no. 1, pp. 46-50, 2011.

[6] C. Li, M. Wu, J. Wang et al., "A population-based study on the risks of cervical lesion and human papillomavirus infection among women in Beijing, People's Republic of China," Cancer Epidemiology Biomarkers and Prevention, vol. 19, no. 10, pp. 2655-2664, 2010. 
[7] R. F. Madeiro Da Costa, W. De Souza, M. Benchimol, J. F. Alderete, and J. A. Morgado-Díaz, "Trichomonas vaginalis perturbs the junctional complex in epithelial cells," Cell Research, vol. 15, no. 9, pp. 704-716, 2005.

[8] J. W. Barrington, D. Linton, A. O'Leary, A. Blackwell, J. Brick, and J. P. Calvert, "Anaerobic (bacterial) vaginosis and premalignant disease of the cervix," Journal of Obstetrics and Gynaecology, vol. 17, no. 4, pp. 383-385, 1997.

[9] A. M. E. Roeters, M. E. Boon, M. Van Haaften, F. Vernooij, T. R. Bontekoe, and A. P. M. Heintz, "Inflammatory events as detected in cervical smears and squamous intraepithelial lesions," Diagnostic Cytopathology, vol. 38, no. 2, pp. 85-93, 2010.

[10] Q. Dai, L. Hu, Y. Jiang et al., "An epidemiological survey of bacterial vaginosis, vulvovaginal candidiasis and trichomoniasis in the Tibetan area of Sichuan Province, China," European Journal of Obstetrics Gynecology and Reproductive Biology, vol. 150, no. 2, pp. 207-209, 2010.

[11] S. Ganguly and A. P. Mitchell, "Mucosal biofilms of Candida albicans," Current Opinion in Microbiology, vol. 14, no. 4, pp. 380-385, 2011.

[12] N. Muñoz, X. Castellsagué, A. B. de González, and L. Gissmann, "Chapter 1: HPV in the etiology of human cancer," Vaccine, vol. 24, no. 3, pp. S1-S10, 2006.

[13] Z. Chen, M. Schiffman, R. Herrero et al., "Evolution and taxonomic classification of human papillomavirus 16 (HPV16)related variant genomes: HPV31, HPV33, HPV35, HPV52, HPV58 and HPV67," PLoS ONE, vol. 6, no. 5, Article ID e20183, 2011.

[14] N. T. Brewer and P. L. Reiter, "Re: impact of human papillomavirus (HPV)-6/11/16/18 vaccine on all HPV-associated genital diseases in young women," Journal of the National Cancer Institute, vol. 102, no. 19, article 1517, 2010.

[15] M. Odida, S. Sandin, F. Mirembe, B. Kleter, W. Quint, and E. Weiderpass, "HPV types, HIV and invasive cervical carcinoma risk in Kampala, Uganda: a case-control study," Infectious Agents and Cancer, vol. 6, no. 1, article 8, 2011.

[16] B. Lu, A. Kumar, X. Castellsagué, and A. R. Giuliano, "Efficacy and safety of prophylactic vaccines against cervical HPV infection and diseases among women: a systematic review \& meta-analysis," BMC Infectious Diseases, vol. 11, article 13, 2011.

[17] K. Shigehara, S. Kawaguchi, T. Sasagawa et al., "Prevalence of genital Mycoplasma, Ureaplasma, Gardnerella, and human papillomavirus in Japanese men with urethritis, and risk factors for detection of urethral human papillomavirus infection," Journal of Infection and Chemotherapy, vol. 17, no. 4, pp. 487-492, 2011.

[18] T. R. Jenkins, K. Hoover, B. Gleason, L. L. Joiner, R. Holley, and H. Greer, "Discussion: "Bacterial vaginosis and STI risk" by Allsworth et al," American Journal of Obstetrics and Gynecology, vol. 205, no. 2, pp. e1-e4, 2011.

[19] S. S. Olmsted, L. A. Meyn, L. C. Rohan, and S. L. Hillier, "Glycosidase and proteinase activity of anaerobic gram-negative bacteria isolated from women with bacterial vaginosis," Sexually Transmitted Diseases, vol. 30, no. 3, pp. 257-261, 2003.

[20] R. F. Lamont, J. D. Sobel, R. A. Akins et al., "The vaginal microbiome: new information about genital tract flora using molecular based techniques," BJOG, vol. 118 , no. 5, pp. 533$549,2011$.

[21] C. H. Livengood, "Bacterial vaginosis: an overview for 2009," Reviews in Obstetrics \& Gynecology, vol. 2, pp. 28-37.
[22] Z. Krzemiński, J. Kalinka, and E. Brzezińska-Błaszczyk, "Correlation between levels of selected cytokines in cervicovaginal fluid of women with abnormal vaginal bacterial flora," Medycyna Doświadczalna i Mikrobiologia, vol. 57, no. 3, pp. 327-333, 2005.

[23] H. Verstraelen and R. Verhelst, "Bacterial vaginosis: an update on diagnosis and treatment," Expert Review of Anti-Infective Therapy, vol. 7, no. 9, pp. 1109-1124, 2009.

[24] R. Bologno, Y. M. Díaz, M. C. Giraudo et al., "Importance of studying the balance of vaginal content (BAVACO) in the preventive control of sex workers," Revista Argentina de Microbiologia, vol. 43, no. 4, pp. 246-250, 2011.

[25] J. Koshiol, L. Lindsay, J. M. Pimenta, C. Poole, D. Jenkins, and J. S. Smith, "Persistent human papillomavirus infection and cervical neoplasia: a systematic review and meta-analysis," American Journal of Epidemiology, vol. 168, no. 2, pp. 123-137, 2008.

[26] J. M. Marrazzo, K. K. Thomas, K. Agnew, and K. Ringwood, "Prevalence and risks for bacterial vaginosis in women who have sex with women," Sexually Transmitted Diseases, vol. 37, no. 5, pp. 335-339, 2010.

[27] N. S. Botelho, S. B. de Paula, L. A. Panagio, P. Pinge-Filho, L. M. Yamauchi, and S. F. Yamada-Ogatta, "Candida species Isolated from urban bats of Londrina-Paraná, Brazil and their potential virulence," Zoonoses and Public Health, vol. 59, no. 1, pp. 16-22, 2011.

[28] M. K. Engberts, C. F. W. Vermeulen, B. S. M. Verbruggen, M. Van Haaften, M. E. Boon, and A. P. M. Heintz, "Candida and squamous (pre)neoplasia of immigrants and Dutch women as established in population-based cervical screening," International Journal of Gynecological Cancer, vol. 16, no. 4, pp. 15961600, 2006.

[29] E. F. Murta, M. A. Souza, E. Araújo Júnior, and S. J. Adad, "Incidence of Gardnerella vaginalis, Candida sp and human papilloma virus in cytological smears," Sao Paulo Medical Journal, vol. 118, no. 4, pp. 105-108, 2000.

[30] P. D. Wang and R. S. Lin, "Epidemiologic differences between candidial and trichomonal infections as detected in cytologic smears in Taiwan," Public Health, vol. 109, no. 6, pp. 443-450, 1995.

[31] R. N. Chakrabarti, K. Dutta, T. Sarkhel, and S. Maity, "Cytologic evidence of the association of different infective lesions with dysplastic changes in the uterine cervix," European Journal of Gynaecological Oncology, vol. 13, no. 5, pp. 398-402, 1992.

[32] M. K. Engberts, B. S. Verbruggen, M. E. Boon, M. van Haaften, and A. P. Heintz, "Candida and dysbacteriosis: a cytologic, population-based study of 100,605 asymptomatic women concerning cervical carcinogenesis," Cancer, vol. 111, no. 5, pp. 269-274, 2007.

[33] D. H. Watts, G. Springer, H. Minkoff et al., "The occurrence of vaginal infections among HIV-infected and high-risk HIVuninfected women: longitudinal findings of the women's interagency HIV study," Journal of Acquired Immune Deficiency Syndromes, vol. 43, no. 2, pp. 161-168, 2006.

[34] S. R. Leon, K. A. Konda, K. T. Bernstein et al., "Trichomonas vaginalis infection and associated risk factors in a sociallyMarginalized female population in coastal peru," Infectious Diseases in Obstetrics and Gynecology, vol. 2009, Article ID 752437, 6 pages, 2009.

[35] A. Storti-Filho, P. C. Souza, F. Chassot et al., "Association of public versus private health care utilization and prevalence of Trichomonas vaginalis in Maringá, Paraná, Brazil," Archives of Gynecology and Obstetrics, vol. 280, no. 4, pp. 593-597, 2009. 
[36] S. Rughooputh and P. Greenwell, "Trichomonas vaginalis: paradigm of a successful sexually transmitted organism," British Journal of Biomedical Science, vol. 62, no. 4, pp. 193-200, 2005.

[37] D. Provenzano, A. Khoshnan, J. F. Alderete, S. Rughooputh, and P. Greenwell, "Trichomonas vaginalis: paradigm of a successful sexually transmitted organism," British Journal of Biomedical Science, vol. 62, no. 4, pp. 193-200, 2005.

[38] M. Y. Zheng, H. L. Zhao, J. P. Di et al., "Association of human papillomavirus infection with other microbial pathogens in gynecology," Zhonghua fu Chan Ke Za Zhi, vol. 45, no. 6, pp. 424-428, 2010.

[39] R. Arroyo and J. F. Alderete, "Two Trichomonas vaginalis surface proteinases bind to host epithelial cells and are related to levels of cytoadherence and cytotoxicity," Archives of Medical Research, vol. 26, no. 3, pp. 279-285, 1995.

[40] A. Wang, C. C. Wang, and J. F. Alderete, "Trichomonas vaginalis phenotypic variation occurs only among trichomonads infected with the double-stranded RNA virus," Journal of Experimental Medicine, vol. 166, no. 1, pp. 142-150, 1987.

[41] M. E. Scheurer, G. Tortolero-Luna, and K. Adler-Storthz, "Human papillomavirus infection: biology, epidemiology, and prevention," International Journal of Gynecological Cancer, vol. 15, no. 5, pp. 727-746, 2005.

[42] S. M. Garland, S. N. Tabrizi, S. Chen, C. Byambaa, and K. Davaajav, "Prevalence of sexually transmitted infections (Neisseria gonorrhoeae, Chlamydia trachomatis, Trichomonas vaginalis and human papillomavirus) in female attendees of a sexually transmitted diseases clinic in Ulaanbaatar, Mongolia," Infectious Diseases in Obstetrics and Gynecology, vol. 9, no. 3, pp. 143-146, 2001.

[43] J. C. Noël, I. Fayt, M. R. Romero Munoz, P. Simon, and C. Engohan-Aloghe, "High prevalence of high-risk human papillomavirus infection among women with Trichomonas vaginalis infection on monolayer cytology," Archives of Gynecology and Obstetrics, vol. 282, no. 5, pp. 503-505, 2010.

[44] C. E. Depuydt, E. Leuridan, P. Van Damme, J. Bogers, A. J. Vereecken, and G. G. G. Donders, "Epidemiology of Trichomonas vaginalis and human papillomavirus infection detected by real-time PCR in flanders," Gynecologic and Obstetric Investigation, vol. 70, no. 4, pp. 273-280, 2010. 


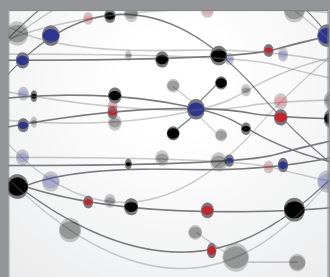

The Scientific World Journal
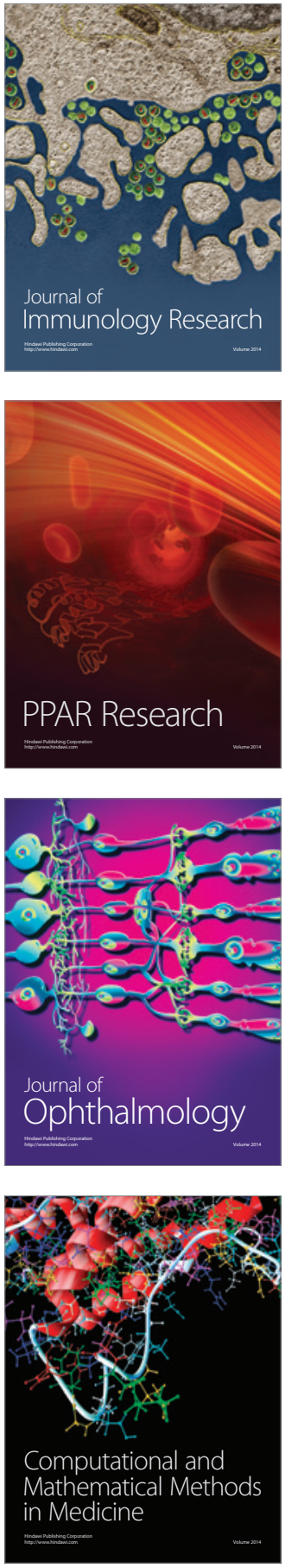

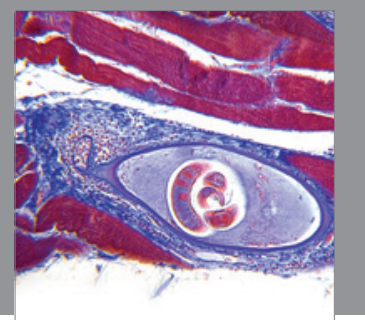

Gastroenterology

Research and Practice
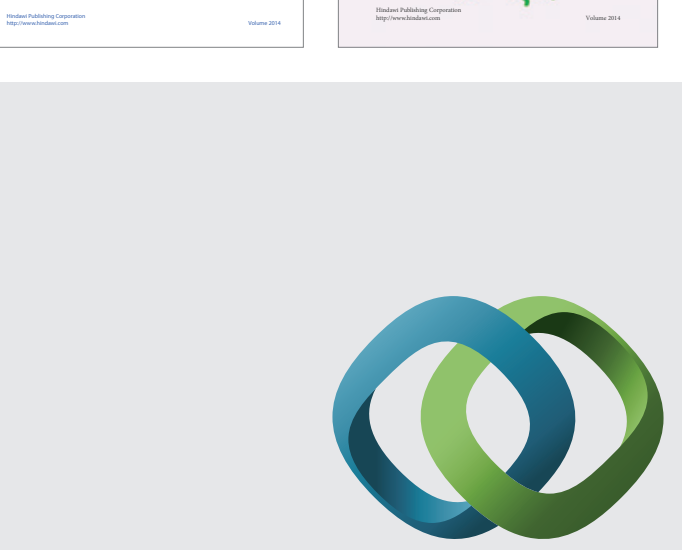

\section{Hindawi}

Submit your manuscripts at

http://www.hindawi.com
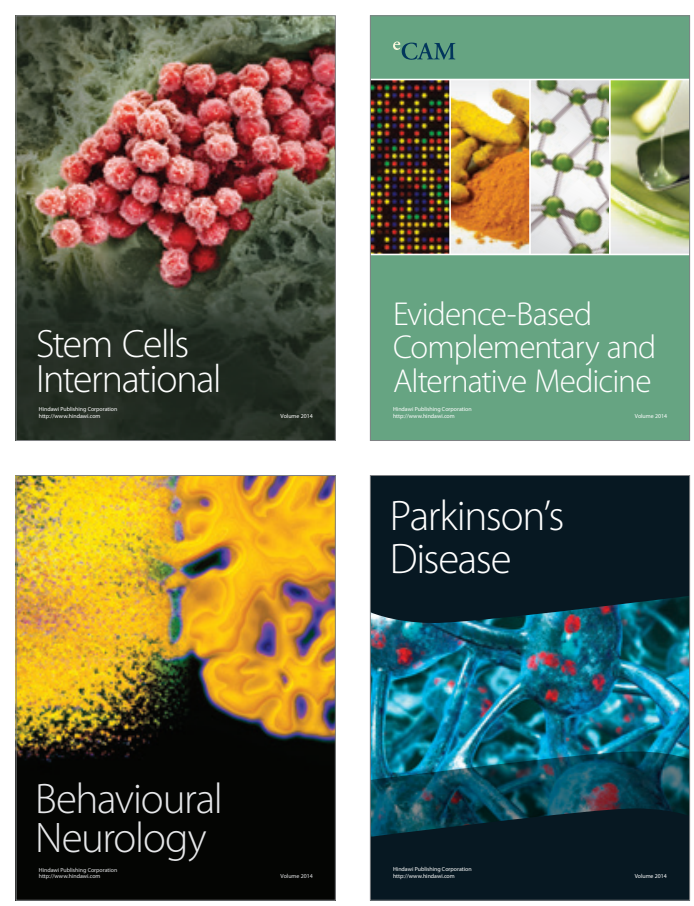

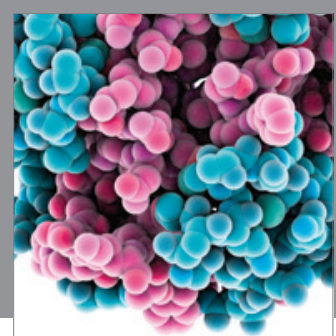

Journal of
Diabetes Research

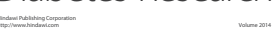

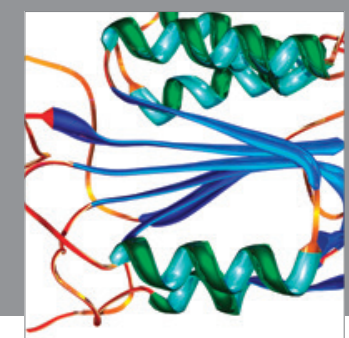

Disease Markers
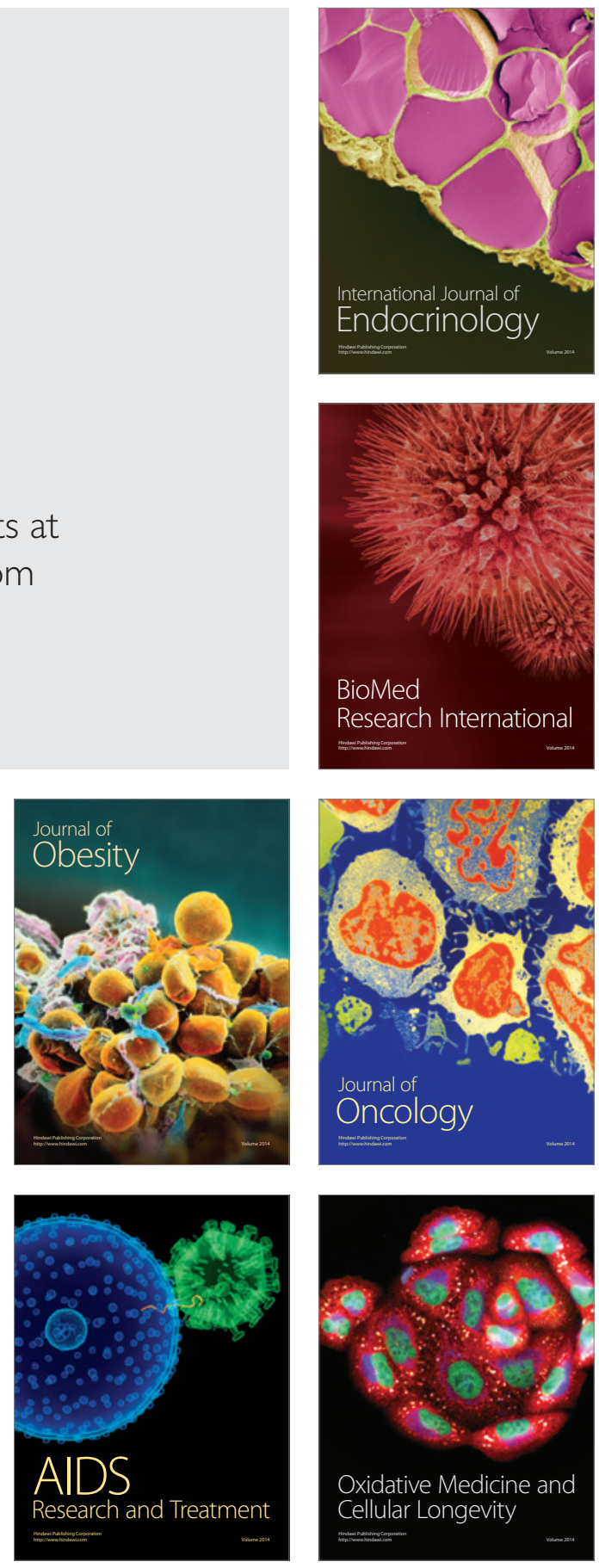